\title{
Correction to: On Guay's Evaluation Map for Affine Yangians
}

\author{
Ryosuke Kodera ${ }^{1}$
}

Published online: 4 December 2020

(C) Springer Nature B.V. 2020

Correction to: Algebras and Representation Theory https://doi.org/10.1007/s10468-019-09945-w

After this paper was published online, an error was found. There was an error in the proof of Theorem 3.1 (main theorem) in the earlier version, and consequently it was wrong as stated. We need to correct the definition of the affine Lie algebra $\hat{\mathfrak{g l}}_{N}$. More precisely we need to modify the defining relation of the diagonal Heisenberg part of $\hat{\mathfrak{g l}}_{N}$. Then we can show that there exists an algebra homomorphism from the affine Yangian to a completion of the universal enveloping algebra of $\hat{\mathfrak{g l}}_{N}$ as desired. The condition among parameters and the explicit form of the evaluation map need not to be changed. The actual change in the proof of Theorem 3.1 concerns the relation (3.2), namely Lemma 3.3. Let us summarize corrections made in this version: the definition of $\hat{\mathfrak{g l}}{ }_{N}$ in Section 2.2; the values of $\left[A_{i}, A_{j}\right]$ and $\left[B_{i}, A_{j}\right]$ in Lemma 3.3 along with its proof; Remark 4.2.

The corrected version is available on arXiv: 1806.09884 .

\subsection{Affine Lie Algebra $\hat{\mathfrak{g l}}_{N}$}

Section 2.2 will be modified as follows.

Let $\mathfrak{g l}_{N}$ be the complex general linear Lie algebra consisting of $N \times N$ matrices. We denote by $E_{i, j}$ the matrix unit with $(i, j)$-th entry 1 . The indices $i, j$ of $E_{i, j}$ are regarded

The online version of the original article can be found at https://doi.org/10.1007/s10468-019-09945-w.

The author moved to Chiba University from Kobe University last September 2020. His correct affiliation and email address is presented in this correction article.

Ryosuke Kodera

kodera@math.s.chiba-u.ac.jp

1 Department of Mathematics and Informatics, Graduate School of Science, Chiba University, Chiba 263-8522, Japan 
as elements of $\mathbb{Z} / N \mathbb{Z}$. The transpose of an element $X$ of $\mathfrak{g l}_{N}$ is denoted by ${ }^{t} X$. Put $\mathbf{1}=$ $\sum_{i=1}^{N} E_{i, i}$ and $\mathfrak{a}=\mathbb{C} 1$. We have a decomposition

$$
\mathfrak{g l}_{N}=\mathfrak{s l}_{N} \oplus \mathfrak{a} \text {. }
$$

Let $\hat{\mathfrak{s l}}_{N}=\mathfrak{s l}_{N} \otimes \mathbb{C}\left[t, t^{-1}\right] \oplus \mathbb{C} c$ be the affine Lie algebra whose Lie bracket is given by $\left[X \otimes t^{r}, Y \otimes t^{s}\right]=[X, Y] \otimes t^{r+s}+r \delta_{r+s, 0} \operatorname{tr}(X Y) c, \quad c$ is central.

Let $\hat{\mathfrak{a}}=\mathfrak{a} \otimes \mathbb{C}\left[t, t^{-1}\right] \oplus \mathbb{C} c^{\prime}$ be the Heisenberg Lie algebra whose Lie bracket is given by

$$
\left[\mathbf{1} \otimes t^{r}, \mathbf{1} \otimes t^{s}\right]=r \delta_{r+s, 0} N c^{\prime}, \quad c^{\prime} \text { is central. }
$$

Define two kinds of the affine Lie algebra $\hat{\mathfrak{g l}} \mathrm{l}_{N}$ by

$$
\hat{\mathfrak{g l}}_{N}^{(+)}=\left(\hat{\mathfrak{s l}}_{N} \oplus \hat{\mathfrak{a}}\right) /\left(c^{\prime}-(c+N)\right), \quad \hat{\mathfrak{g l}}_{N}^{(-)}=\left(\hat{\mathfrak{s l}}_{N} \oplus \hat{\mathfrak{a}}\right) /\left(c^{\prime}-(c-N)\right) .
$$

Then we have

$$
\left[E_{i, i} \otimes t^{r}, E_{j, j} \otimes t^{s}\right]=r \delta_{r+s, 0}\left(\delta_{i, j} c+1\right) \quad \text { in } \quad \hat{\mathfrak{g l}}_{N}^{(+)}
$$

and

$$
\left[E_{i, i} \otimes t^{r}, E_{j, j} \otimes t^{s}\right]=r \delta_{r+s, 0}\left(\delta_{i, j} c-1\right) \quad \text { in } \quad \hat{\mathfrak{g l}}_{N}^{(-)} .
$$

In the sequel, the symbol $\hat{\mathfrak{g l}}_{N}$ denotes both $\hat{\mathfrak{g l}}_{N}^{(+)}$and $\hat{\mathfrak{g l}}_{N}^{(-)}$unless otherwise stated.

We denote the element $X \otimes t^{s}$ by $X(s)$. We set

$$
\begin{aligned}
& x_{0}^{+}=E_{N, 1}(1), \quad x_{0}^{-}=E_{1, N}(-1), \quad h_{0}=E_{N, N}-E_{1,1}+c, \\
& x_{i}^{+}=E_{i, i+1}, \quad x_{i}^{-}=E_{i+1, i}, \quad h_{i}=E_{i, i}-E_{i+1, i+1} \quad(i \neq 0) .
\end{aligned}
$$

Let $\hat{\mathfrak{n}}_{ \pm}$be the Lie subalgebras of $\hat{\mathfrak{g l}}_{N}$ generated by $x_{i}^{ \pm}(i \in \mathbb{Z} / N \mathbb{Z})$ and $\mathbf{1}(s)( \pm s>0)$. That is,

$$
\hat{\mathfrak{n}}_{+}=\bigoplus_{\substack{i<j \\ s \geq 0}} \mathbb{C} E_{i, j}(s) \oplus \bigoplus_{\substack{i \geq j \\ s>0}} \mathbb{C} E_{i, j}(s), \quad \hat{\mathfrak{n}}_{-}=\bigoplus_{\substack{i>j \\ s \leq 0}} \mathbb{C} E_{i, j}(s) \oplus \bigoplus_{\substack{i \leq j \\ s<0}} \mathbb{C} E_{i, j}(s) .
$$

Let $\hat{\mathfrak{h}}$ be the Cartan subalgebra generated by $h_{i}(i \in \mathbb{Z} / N \mathbb{Z})$ and $\mathbf{1}$.

Let $\omega_{U}$ be the algebra anti-automorphism of $U\left(\hat{\mathfrak{g l}}_{N}\right)$ defined by $\omega_{U}(X(s))={ }^{t} X(-s)$ and $\omega_{U}(c)=c$. We denote by $\mu_{U}$ the algebra anti-isomorphism from $U\left(\hat{g r l}_{N}^{(+)}\right)$to $U\left(\hat{g r l}_{N}^{(-)}\right)$ induced from the assignment $X$ in $\hat{\mathfrak{g l}}_{N}^{(+)} \mapsto-X$ in $\hat{\mathfrak{g l}}_{N}^{(-)}$. The restriction of $\mu_{U}$ to $\hat{\mathfrak{s l}}_{N}$ gives an algebra anti-automorphism of $\hat{\mathfrak{s l}}_{N}$. The anti-isomorphism $\mu$ defined in the previous subsection is an extension of the restriction of $\mu_{U}$ to $\hat{\mathfrak{s l}}_{N}$.

We define gradings of $\hat{\mathfrak{n}}_{ \pm}$by $\operatorname{deg} X(s)=s$. Then $U\left(\hat{\mathfrak{n}}_{ \pm}\right)$become graded algebras. We denote by $U\left(\hat{\mathfrak{n}}_{ \pm}\right)[s]$ the degree $s$ components. Let us introduce completions of $U\left(\hat{\mathfrak{g l}}_{N}^{(+)}\right)$ and $U\left(\hat{\mathfrak{g l}}_{N}^{(-)}\right)$.

Definition 2.5 We define completions $\left.U(\hat{\mathfrak{g l}})_{N}\right)_{\text {comp, },}$ and $\left.U(\hat{\mathfrak{g l}})_{N}\right)_{\text {comp,- }}$ of $U\left(\hat{\mathfrak{g l}}{ }_{N}^{(+)}\right)$and $U\left(\hat{\mathfrak{g l}}_{N}^{(-)}\right)$, respectively, as follows:

$$
\begin{aligned}
& \left.U(\hat{\mathfrak{g l}})_{N}\right)_{\text {comp },+}=\bigoplus_{k \in \mathbb{Z}} \prod_{\substack{r, s \geq 0 \\
s-r=k}}\left(U\left(\hat{\mathfrak{n}}_{-}\right)[-r] \otimes U(\hat{\mathfrak{h}}) \otimes U\left(\hat{\mathfrak{n}}_{+}\right)[s]\right), \\
& U\left(\hat{\mathfrak{g} l_{N}}\right)_{\mathrm{comp},-}=\bigoplus_{k \in \mathbb{Z}} \prod_{\substack{r, s \geq 0 \\
r-s=k}}\left(U\left(\hat{\mathfrak{n}}_{+}\right)[r] \otimes U(\hat{\mathfrak{h}}) \otimes U\left(\hat{\mathfrak{n}}_{-}\right)[-s]\right) .
\end{aligned}
$$


Both $\left.U(\hat{\mathfrak{g l}})_{N}\right)_{\text {comp, }+}$ and $U\left(\hat{\mathfrak{g l}}_{N}\right)_{\text {comp,- }}$ have natural algebra structures which contain $U\left(\hat{\mathfrak{g l}}_{N}^{(+)}\right)$and $U\left(\hat{\mathfrak{g l}}_{N}^{(-)}\right)$as subalgebras, respectively. Moreover the anti-automorphism $\omega_{U}$ of $U\left(\hat{\mathfrak{g l}} \mathrm{l}_{N}\right)$ extends to the completions and the anti-isomorphism $\mu_{U}: U\left(\hat{\mathfrak{g l}}_{N}^{(+)}\right) \rightarrow U\left(\hat{\mathfrak{g l}}_{N}^{(-)}\right)$ extends to an algebra anti-isomorphism

$$
\left.\left.\mu_{U}: U(\hat{\mathfrak{g l}})_{N}\right)_{\text {comp },+} \rightarrow U(\hat{\mathfrak{g l}})_{N}\right)_{\text {comp },-} .
$$

\subsection{Main Theorem}

After the modification of the definition of $\hat{\mathfrak{g l}}_{N}$ as above, the statements of our main theorems Theorem 3.1 and 3.8 remain unchanged.

We give a correction of Lemma 3.3. It is only a necessary modification for the proof of Theorem 3.1. Lemma 3.3 will be modified as follows.

Lemma 3.3 We have

$$
\begin{gathered}
{\left[A_{i}, A_{j}\right]=\sum_{\substack{r, s>0 \\
r>s}} \sum_{\substack{i \\
+}}^{i}\left(E_{k, i}(r) E_{i, j}(s-r) E_{j, k}(-s)-E_{k, j}(s) E_{j, i}(r-s) E_{i, k}(-r)\right)} \\
{\left[E_{i, i}(r) E_{j, j}(-r)-E_{j, j}(r) E_{i, i}(-r)\right),} \\
{\left[A_{j}\right]=0,}
\end{gathered}
$$

$$
\begin{aligned}
& {\left[B_{i}, A_{j}\right]} \\
& =\sum_{r, s \geq 0}\left(\sum_{k=1}^{i}\left(-E_{k, i}(r+s+1) E_{i, j}(-r-1) E_{j, k}(-s)+E_{k, j}(s) E_{j, i}(r+1) E_{i, k}(-r-s-1)\right)\right. \\
& \left.\quad+\sum_{k=j+1}^{N}\left(-E_{k, i}(r+1) E_{i, j}(s) E_{j, k}(-r-s-1)+E_{k, j}(r+s+1) E_{j, i}(-s) E_{i, k}(-r-1)\right)\right) \\
& \quad+\sum_{r>0} r\left(-E_{i, i}(r) E_{j, j}(-r)+E_{j, j}(r) E_{i, i}(-r)\right), \\
& {\left[B_{i}, B_{j}\right]} \\
& =\sum_{\substack{r, s \geq 0 \\
r \leq s}} \sum_{\substack{k=j+1 \\
\text { ind }}}^{N}\left(E_{k, i}(r+1) E_{i, j}(s-r) E_{j, k}(-s-1)-E_{k, j}(s+1) E_{j, i}(r-s) E_{i, k}(-r-1)\right) .
\end{aligned}
$$

Proof We need to correct computations of $\left[A_{i}, A_{j}\right]$ and $\left[B_{i}, A_{j}\right]$.

We compute $\left[A_{i}, A_{j}\right]$ as

$$
\begin{aligned}
{\left[A_{i}, A_{j}\right]=} & \sum_{r, s \geq 0} \sum_{k=1}^{i}\left(E_{k, j}(r+s) E_{i, k}(-r) E_{j, i}(-s)+E_{k, i}(r) E_{i, j}(s-r) E_{j, k}(-s)\right. \\
& \left.-E_{k, j}(s) E_{j, i}(r-s) E_{i, k}(-r)-E_{i, j}(s) E_{k, i}(r) E_{j, k}(-r-s)\right) \\
& +\sum_{r>0} r\left(E_{i, i}(r) E_{j, j}(-r)-E_{j, j}(r) E_{i, i}(-r)\right) .
\end{aligned}
$$

Then, a further computation shows the first identity. 
A direct computation shows

$$
\begin{aligned}
& {\left[B_{i}, A_{j}\right]} \\
& =\sum_{r, s \geq 0}\left(\sum_{k=i+1}^{N} E_{k, j}(r+s+1) E_{i, k}(-r-1) E_{j, i}(-s)-\sum_{k=1}^{j} E_{k, i}(r+s+1) E_{i, j}(-r-1) E_{j, k}(-s)\right. \\
& +\sum_{k=i+1}^{j}\left(E_{k, i}(r+1) E_{i, j}(s-r-1) E_{j, k}(-s)-E_{k, j}(s) E_{j, i}(r-s+1) E_{i, k}(-r-1)\right) \\
& \left.+\sum_{k=1}^{j} E_{k, j}(s) E_{j, i}(r+1) E_{i, k}(-r-s-1)-\sum_{k=i+1}^{N} E_{i, j}(s) E_{k, i}(r+1) E_{j, k}(-r-s-1)\right) .
\end{aligned}
$$

The sums of the terms containing

$$
E_{k, i}\left(a_{1}\right), E_{i, j}\left(a_{2}\right), E_{j, k}\left(a_{3}\right)\left(a_{1}, a_{2}, a_{3} \in \mathbb{Z}\right), \quad E_{k, j}\left(a_{1}\right), E_{j, i}\left(a_{2}\right), E_{i, k}\left(a_{3}\right)\left(a_{1}, a_{2}, a_{3} \in \mathbb{Z}\right)
$$

are

$$
\begin{aligned}
& \sum_{r, s \geq 0}\left(-\sum_{k=1}^{i} E_{k, i}(r+s+1) E_{i, j}(-r-1) E_{j, k}(-s)-\sum_{k=j+1}^{N} E_{k, i}(r+1) E_{i, j}(s) E_{j, k}(-r-s-1)\right. \\
&\left.+\sum_{k=i+1}^{N} E_{k, j}(r+s+1) E_{j, k}(-r-s-1)\right)-\sum_{r>0} r E_{i, i}(r) E_{j, j}(-r), \\
& \sum_{r, s \geq 0}\left(\sum_{k=j+1}^{N} E_{k, j}(r+s+1) E_{j, i}(-s) E_{i, k}(-r-1)+\sum_{k=1}^{i} E_{k, j}(s) E_{j, i}(r+1) E_{i, k}(-r-s-1)\right. \\
&\left.\quad-\sum_{k=i+1}^{N} E_{k, j}(r+s+1) E_{j, k}(-r-s-1)\right)+\sum_{r>0} r E_{j, j}(r) E_{i, i}(-r),
\end{aligned}
$$

respectively. Hence the third identity holds.

\section{Evaluation Modules}

In Section 4, the symbol $\hat{\mathfrak{g l}}_{N}$ denotes $\hat{\mathfrak{g l}}_{N}^{(+)}$except in Remark 4.2. Remark 4.2 will be modified as follows.

Remark 4.2 In [9], the author proves that the image of Guay's evaluation map ev in $\left.U(\hat{\mathfrak{g l}})_{N}\right)_{\text {comp,- contains }} \hat{\mathfrak{g l}}_{N}^{(-)}$under the assumption $\varepsilon_{2} \neq 0$. This result implies that the image of $\mathrm{ev}_{\alpha}^{+}$in $\left.U(\hat{\mathfrak{g l}})_{N}\right)_{\text {comp, },}$ contains $\hat{\mathfrak{g l}}_{N}^{(+)}$under the assumption $\varepsilon_{1} \neq 0$. Hence the $Y\left(\hat{\mathfrak{s l}}_{N}\right)$-module $L(\Lambda, \alpha)$ is irreducible when $\varepsilon_{1} \neq 0$. We do not use this fact in the proof given below.

Acknowledgments The author thanks to Mamoru Ueda for pointing out an error in the published version of this paper.

Publisher's Note Springer Nature remains neutral with regard to jurisdictional claims in published maps and institutional affiliations. 